\title{
THREE CLEAN PRODUCTS FROM CO-MINGLED WASTE USING A NOVEL HYDRODYNAMIC SEPARATOR
}

\author{
L.B. ESTEBAN ${ }^{1}$, J.S. SHRIMPTON ${ }^{1}$, P. ROGERS ${ }^{2} \&$ R. INGRAM ${ }^{2}$ \\ ${ }^{1}$ Southampton University, UK \\ ${ }^{2}$ Aquavitrum, UK.
}

\begin{abstract}
Mixed municipal solid waste currently landfilled contains a high percentage of packaging glass, varying from $33 \%$ to $80 \%$ by weight according to several sample characterizations, due to the lack of high throughput separation technology. The hydrodynamic separator proposed is a closed-loop device developed to separate co-mingled waste into plastics, glass and other dense particles and organic sludge. The glass and other dense material stream is cleaned by the system, permitting efficient downstream optical sorting to take out metals and ceramics and, if required, glass sorted by colour. The plastics and the organic sludge are separate, processable waste streams. As the solid waste is introduced in the separator, the action of water jets located on the ramps of a fixed sinusoidal-shape bottom and the presence of hydrofoils at the upper part of the tank produce a flow pattern that lead plastics towards its collection point on the surface, while glass and ceramics are settled to the bottom of the tank and transported to the extraction point. Organics and other fine particles are obtained from lamellas, before reintroducing the clarified fluid into the flow loop. The sludge obtained from this process is suitable for feedstock to Anaerobic Digestion processes. In the present paper the equipment and the methodology is described and the physical principles of the separation process are explained. Results from a full scale trial designed to process 9.7 tonnes per hour at a municipal UK waste site operating in Nov 2015 - Feb 2016 are presented.
\end{abstract}

Keywords: co-mingled, fluid-dynamics, glass, plastic, recycling, separation, turbulence.

\section{INTRODUCTION}

Solid waste management is a term that embraces a wide variety of operations; from waste collecting techniques in urban areas to complex techniques to reduce the waste disposal. This study is focused on the development of a technology capable of taking household waste and separating plastics from glass while obtaining a third product suitable for its direct usage as feedstock to organic digestion processes.

The first serious concern about what today is known as municipal solid waste was in Athens in 320 B.C. when a law forbidding throwing waste onto streets was established [1]. This concern regarding waste management has evolved together with human societies, improving the waste management standards. After the first refuse incinerator was built in England in 1874, a rapid growth of this practise was experienced, $15 \%$ of the major American cities adopted it at the beginning of the 20th century [2].

Nevertheless, it has become evident that improper incineration of solid waste and open dumping cause severe pollution problems such as intense air pollution due to particulate matter, carbon monoxide and hydrocarbons release and water pollution due to leakages from dumpsites. 
Additionally, the rapid increase in the amount of waste generated worldwide has forced governments and institutions to tackle this issue. Thus, solid waste management plants emphasize the practice of recycling and waste sorting in the vast majority of developed countries, keeping the incineration and land disposal as a last alternative.

The U.K. produced 5.7 million tonnes of dry recycling from households in 2013 [3], glass being the second product in terms of weight percentage, representing $19 \%$ of the overall. Recycling approximately the $68 \%$ of packaging glass annual consumption, an outstanding proportion of glass is still contained within the residual waste produced by Material Recovering Facilities (MRF's). This residual glass is currently landfilled or incinerated, representing not only a huge economic cost but a problem to the Environment Agency. Due to the toughening of the landfill gate fees, the improvement in the waste management that this technology provides will lead to an impressive cost savings.

The proposed hydrodynamic separator improves the recovery rate of packaging glass in the U.K. from extremely dirty feedstock's and also as a pre-processing step prior to optical sorting, since they need clean feed. Furthermore, plastics extracted from the initial waste product are collected and can be sent to produce Refuse Derived Fuel. In addition, the use of optical sorters as an initial separation process is not preferred since they work best on a relatively narrow size fraction and, the high initial capital investment and operational cost limit their viability.

The usage of this technique also reduces the complexity of the latter optical sorting technique, since conflicting particles of plastic such as CD cases are rarely present in the output glass stream. Besides, the cleanness of the end product helps the optical sorting process if it would be required.

\section{PRINCIPLES FOR PARTICLE SEPARATION}

The hydrodynamic separator is inspired by the behaviour of large particles of higher density than the fluid phase in natural processes, i.e. river bed sedimentation, which is a function of both particle and flow characteristics. The separation process deals with non-spherical particles with complex dynamic behaviour, and therefore spherical particles will be introduced first.

The particle Reynolds number, the particle shape and the fluid regime are the parameters that affect the separation process the most. In what follows are some comments on their specific influence.

\subsection{Particle Reynolds number}

The behaviour of spherical particles suspended in water is seen to be highly dependent on the Reynolds number $R e_{p}$; parameter used to define the nature of the particle surrounding flow.

$$
R e_{p}=\frac{|\vec{u}-\vec{v}| d_{p}}{v}
$$

The term in absolute value is the difference between the flow velocity $\vec{u}$ and the particle velocity $\vec{v}$, the characteristic length scale $d_{p}$ is the diameter of the sphere and $v$ is the fluid kinematic viscosity. Note that boundary layer laminar flow only exists up to $R e_{p} \approx 10$. Increasing the Reynolds number, the boundary layer becomes turbulent leading to a sustained drag reduction and an increase in the terminal velocity, up to $R e_{p} \approx 10^{3}$. Then the terminal velocity becomes constant before reaching the critical regime, at $\operatorname{Re}_{p} \approx 5 \cdot 10^{4}$, Hoerner [4]. 


\subsection{Particle shape}

When non-spherical particles are considered, the particle characteristic dimension becomes a controversial definition. Different approaches are seen in the literature to tackle this ambiguity; Hölzer and Sommerfeld [5] used a simple correlation formula for the drag coefficient of arbitrary-shaped particle taking into account the particle orientation and sphericity, whereas for non-isometric particles such as needles or cylinders other parameters may be more appropriate to account for the particle shape, i.e. aspect ratio, as proposed by Gabito and Tsouris [6].

The steadiness of the falling is clearly dependent upon the wake of the particle. Then, as the Reynolds number increases and the shape of the particle is modified, the falling behaviour will become time dependent, leading to chaotic motion in extreme cases.

Furthermore, the relaxation time of non-spherical particles strongly depends on the axis about which the particle rotates. The definition of a single response time is not enough to characterize them accurately, as demonstrated by Zhang et al. [7], but it provides a first estimation of the particle behaviour, as shown in section 3 .

\subsection{Fluid Reynolds number}

The fluid Reynolds number $R e_{f}$ is used to characterize the flow regime, i.e. laminar or turbulent flow.

$$
\operatorname{Re}_{f}=\frac{u L}{v}
$$

In this case, the linear dimension $L$ is defined by a characteristic travelled length of the fluid. If $R e_{f} \ll 1$, the flow is deterministic and the solid-liquid interaction could be simplified to particles falling in quiescent fluid [8,9]. However, if $R e_{f} \gg 1$, the fluid becomes turbulent leading to strong particle-fluid interactions. The motion of the particle becomes complex and dependent on the fluid characteristic response time $[10,11]$, since the flow in the proposed separator is both turbulent and involves highly non-spherical particles.

For turbulent flow the Stokes number is used to define the particle to fluid response time [12].

$$
S t=\frac{\tau_{p}}{\tau_{f}}
$$

Extensive research has been carried during the last decades to understand and classify the behaviour of small heavy particles under strong solid-liquid interactions [10-12].

For Stokes numbers smaller than unity, the particles have sufficient time to modify its velocity and they are able to maintain the velocity of the flow that surround them. Conversely, having Stokes numbers greater than unity implies that particles will not be able to modulate their velocity to adjust it to the local flow conditions due to their high inertia. Thus, two particles with a different Stokes number can be separated by the same fluid flow. The Kolmogorov timescale is generally considered as the appropriate turbulence scale to study the solid-liquid interaction of these particles.

When multiphase flows have particle mass fraction $>0.2$, typically considered "two-way" coupled, the flow is said to affect the manner particle moves as well as the particles affect the manner the flow behaves. This reverse effect of the particles over the flow has been extensively investigated in the literature [13, 14]. 
Taking into consideration conclusions extracted along the last decades on small heavy particles, the general trend is to observe an increase in the settling velocity for particles with the first characteristic time close to the integral time scale of fluid turbulence $\tau_{l} \sim \mathrm{L}_{l} / \mathrm{u}^{\prime}$ and the second characteristic period comparable to the Taylor microscale $\tau \lambda \sim \frac{\lambda}{u} \approx \sqrt{15} \lambda_{k}$ [12].

It is believed that when particle motions share their velocity phase with the fluid turbulent motion the drag experienced by the particle is reduced.

Conversely, for dispersed particles that are larger than the Kolmogorov scale, the turbulence scale separation cannot be applied and the details of the flow around the particles should be investigated to understand the particle behaviour, as reviewed by Balachandar and Eaton [15] among others. There is a need of fully resolved experiments or simulations to clarify the mechanisms of solid-liquid coupling for these particles.

\section{CHARACTERISTICS OF THE SYSTEM}

Solid-liquid interactions are the core of the hydrodynamic separator working principle, as shown in Fig. 1. The system can be visualised as three sub-volumes, each with a different particle regime. The estimation of the flow regime is made by comparing the momentum response time of a particle with the particle-particle average collision time.

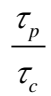

Thus, with ratios higher than unity, particles do not have time to adjust their behaviour to the one imposed by the fluid-dynamic forces, and its motion becomes controlled by subsequent collisions.

At the upper and middle region of the separator, the flow is considered to dilute numbers 1 and 2, respectively, so turbulence characteristics do not change with the particle presence. However, while the hydrofoil region is dominated by the reverse flow and the flow separation that occurs at the rear part of them, the middle region is clearly influenced by the turbulence structures generated in the bottom of the tank. The flow characteristics of this region are of paramount importance in the plastic separation. Contrary to the former regions, the flow exhibits a dense regime at the bottom of the tank number 3 .

The interest of this study resides in the particle dispersion and the influence of turbulence present in the dilute regime. Due to the difficulties associated with the reproduction of the system condition in the laboratory, a drop test and a simple analysis of the turbulence characteristics is carried out.

(1)

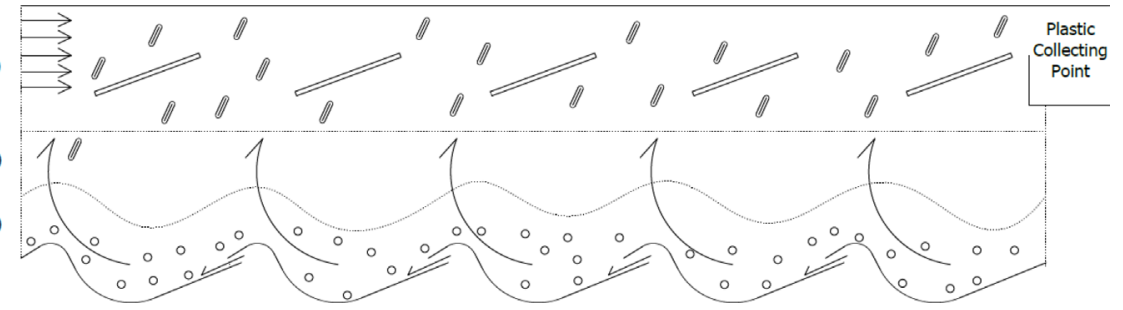

Figure 1: Sketch of the separation section. Broken lines represent the sub-region separation, straight arrows show the base flow jets and curved arrows depict the large scale turbulent structures. Glass (o), Plastics (0) [16]. 
Table 1: Particle response parameters.

\begin{tabular}{lcc}
\hline & Settling vel. & Relax. time \\
\hline Plastic particles & $0.16 \mathrm{~m} / \mathrm{s}$ & $180 \mathrm{~ms}$ \\
Glass particles & $0.29 \mathrm{~m} / \mathrm{s}$ & $50 \mathrm{~ms}$ \\
\hline
\end{tabular}

Using eqn. (5) derived from the general equation of motion and knowing the fluid and particle density, the particle relaxation time $\tau_{\text {peff }}$ is obtained.

$$
v_{\text {set }}=\tau_{p e f f}\left(1-\frac{\rho_{f}}{\rho_{p}}\right) g
$$

The results of the relaxation time in Table 1 show the importance of the particle shape and Reynolds number, since these parameters obtained through preliminary measurements of particle terminal velocity in still fluid are far from the analytical solution of spheres under Stokes flow.

In what follows is a simple analysis of the turbulence characteristics of the tank and the Stokes number of the glass and plastic particles. The turbulence is assumed to be homogeneous and isotropic.

Each separation section is considered to be a closed box of $V=2.7 \mathrm{~m}^{3}$, then it is assumed that all the kinetic energy from the jets becomes turbulent kinetic energy and is dissipated, $P=\in$. Note that energy losses are not considered, i.e wall viscous losses.

Then, the production rate of kinetic energy is given by eqn. (6) as

$$
P=\frac{Q}{V} k \text { Being } k=u_{0}^{2}
$$

Where $Q$ is defined as the water discharged by the $N$ number of jets, their cross-sectional area and the flow velocity $u_{0}$.

The production rate in each section is approximated to,

$$
P=\frac{N \cdot u_{0} \cdot \pi r^{2} \cdot u_{0}^{2}}{V}=\frac{36.15^{3} \cdot \pi \cdot 0.0045^{2}}{2.7}=2.862 \frac{\mathrm{m}^{2}}{\mathrm{~s}^{3}}
$$

The time scales that are considered to control the solid-liquid interaction are the ones associated with the Taylor microscale $\tau_{\lambda}$ and the integral length scale $\tau_{L}$, as suggested by Yang and Shy [12]. The former establishes the limit between eddies whose dynamics is strongly affected by viscosity and the ones that are not while the latter defines the largest scales in the turbulence spectrum.

Following the procedure shown in Pope [17], the following values are obtained, $\tau_{\lambda}=2.3 \cdot 10^{-3} s, \tau_{L}=0.278 s$.

In spite of the strong assumptions considered in this section, the Stokes numbers shown in Table 2 are in agreement with the particle behaviour observed and the theory proposed by Yang and Shy [12]; according to which, glass particles should experience a greater settling velocity due to the smaller phase time difference with the turbulence.

Despite to what has been exposed, the physics of the solid-liquid interaction occurring in the device are unknown due to the irregular shape of particles and the turbulence effects on them. Research is currently underway to give answer to the questions that arise. 
Table 2: Stokes number based on the eddy turnover time of the integral length scale.

\begin{tabular}{ll}
\hline & $S t_{\mathbf{L}}$ \\
\hline Plastic particles & $\sim 6.5$ \\
Glass particles & $\sim 1.8$ \\
\hline
\end{tabular}

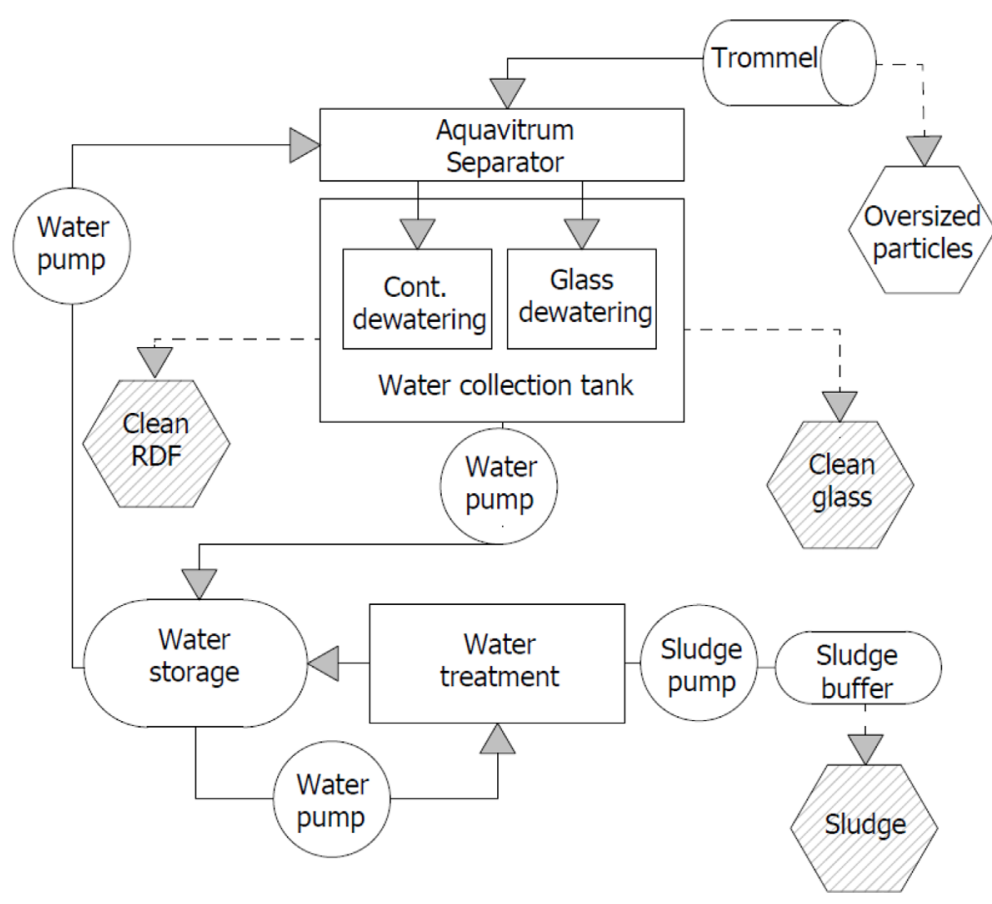

Figure 2: Flow chart of the complete separation process.

\subsection{Materials and methods}

The essence of the equipment is a water tank with three types of sections composing the whole device; the feed chute, the separation zone and the discharge chute.

As it can be seen in Fig. 2, co-mingled waste is fed onto the separator by falling from a mobile trommel (Doppstadt SM518) with a mesh size of $50 \mathrm{~mm}$ in a continuous and steady flow rate. The feeder is located at the beginning of the feed chute to allow particles to go through the whole system, increasing the efficiency of the separation process. Oversize particles that have not passed through the mesh are directly returned to the trommel for a second passing.

The feed chute has a flat surface tilted downwards widely equipped with water jets. The design of the feed chute enables glass and plastic particles to increase its inertia before reaching the first formation of the main section.

The separation zone is composed of modifiable formation shapes located at the bottom, acting as the actual lower tank surface. The proper design of the formation shapes modulates the turbulence and the flow characteristics in accordance with the time scale associated with 
glass particles. A set of five water jets per formation inject water at a chosen pressure to increase the water circulation at the valleys of the formation, ejecting low-density particles away from the wall when they reach the top of the formation. The main pump that feeds the water jets is a Godwin HL200 m.

Once plastic particles are ejected to the far wall region, the severe velocity fluctuations compared with the low-settling velocity of the plastic delay its deposition, keeping them in recirculation in the upper section.

The existence of an inherent reverse flow at the free surface of the water tank together with the presence of hydrofoils - with adjustable tilt, upward, downward, forward and backward motion - lead the plastic particles to the gutters installed at each separation zone. The gutters, located next to the last hydrofoil, collect water and plastics from the free surface. A system of water channels leads the water together with particles to a set of screens that separates effectively water from plastics, see Fig. 3 . While plastics are carried by a moving belt to the plastics deposit, the remaining water is sent to the water storage deposit and then sent to two lamellas where small particles suspended in the flow are removed. The lamellas are tested and show a suspended particle removal of approximately $80 \%$, providing water with $8 \mathrm{ml}$ of suspended solids per $1000 \mathrm{ml}$ in the worst scenario. From this filtering process, a third stream of sludge is obtained; all within a close loop that ensures minimum environmental contamination and maximum water reuse.
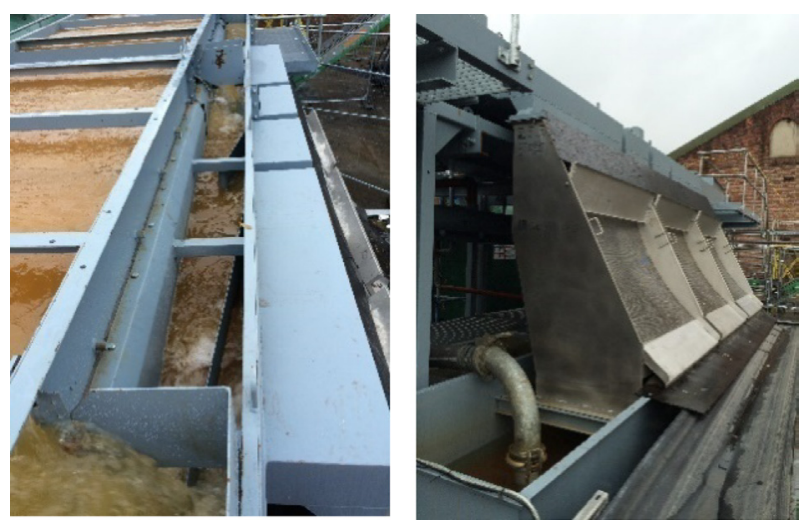

Figure 3: Structure responsible of channelling water to the plastic screens.
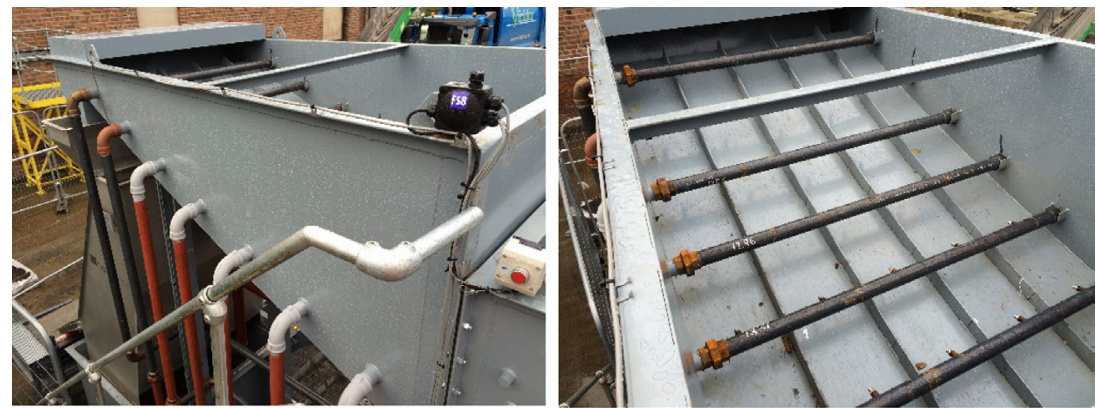

Figure 4: Discharge chute equipped with water jets in span-wise and stream-wise locations. 
After being processed in the separation zone, particles of glass are led to the discharge chute. This last section is composed of a flat plate tilted upwards and another set of water jets, similar to the one present in the feed chute, as shown in Fig. 4. In this case, the jets force every glass particle to move upwards leaving the water tank and reaching the exit of the separator. At that point, glass particles are collected by a moving belt and sent to the glass deposit.

\subsection{Samples}

The experimentation was made with feedstock obtained from two recycling plants; Pearce Recycling Group (Site 1) and Wharf Road (Site 2).

To determine the level of contamination present in the feedstock from Site $1,10 \mathrm{~kg}$ of raw product were firstly split in greater than $10 \mathrm{~mm}$ particles and less than $10 \mathrm{~mm}$ particles by passing it through a $10 \mathrm{~mm}$ screen. The bigger size portion was then spread out onto a clean surface to the manual separation of particles into glass, metals, CSP (ceramics, stones, porcelain and pyro-ceramics), organics, papers (wood, paper and fabrics) and plastics (plastics, films and rubbers); and the latter recording of weights.

The same procedure is followed for the particles smaller than $10 \mathrm{~mm}$; however, in order to reduce the working load of the separation process, the sample is reduced to its $10 \%$ weight approximately.

Once the mass balance of the raw product is recorded, as seen in Fig. 5, the feedstock glass particles are separated according to its size across several grades; less than $2 \mathrm{~mm}, 2-5 \mathrm{~mm}$, $5-10 \mathrm{~mm}, 10-50 \mathrm{~mm}$. For the glass size distribution a portion of $2 \mathrm{~kg}$ is analysed.

\subsection{Working procedure}

Preliminary tests carried out in a scaled model of the hydrodynamic separator showed that the operating variables that influence the separation results the most are the incidence angle of the upper plates and the pressure drop of the water jets.

Table 3: Glass size distribution.

\begin{tabular}{lcccc}
\hline Dimension & $<2 \mathrm{~mm}$ & $2-5 \mathrm{~mm}$ & $5-10 \mathrm{~mm}$ & $10-50 \mathrm{~mm}$ \\
\hline Input (\% weight) & 0.13 & 1.48 & 28.06 & 70.32 \\
Output (\% weight) & 0.03 & 0.75 & 29.32 & 69.90 \\
\hline
\end{tabular}
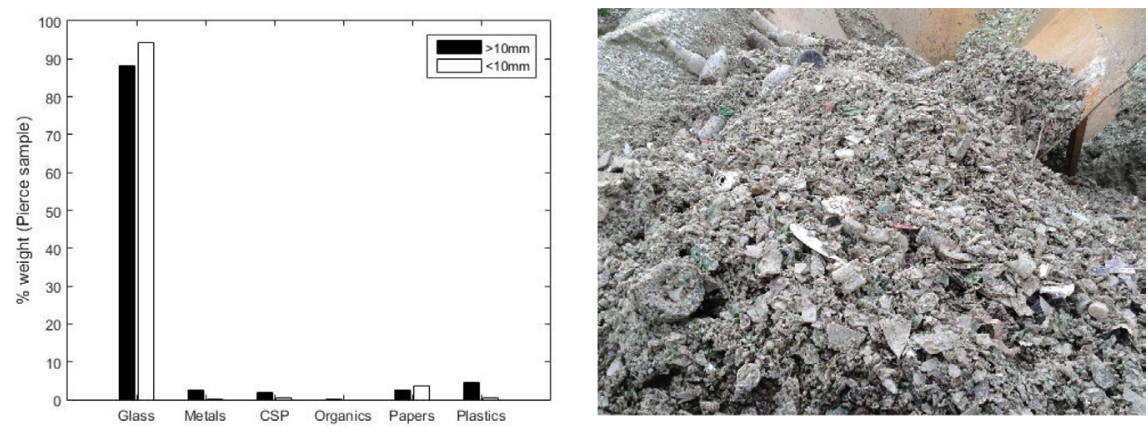

Figure 5: Composition of Site 1 feedstock before the removal of contaminants. 
a)

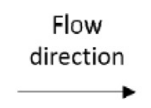

b)

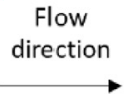

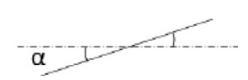

Figure 6: Sketch of the hydrofoil configuration. Positive angle of attack (a), Negative angle of attack (b).
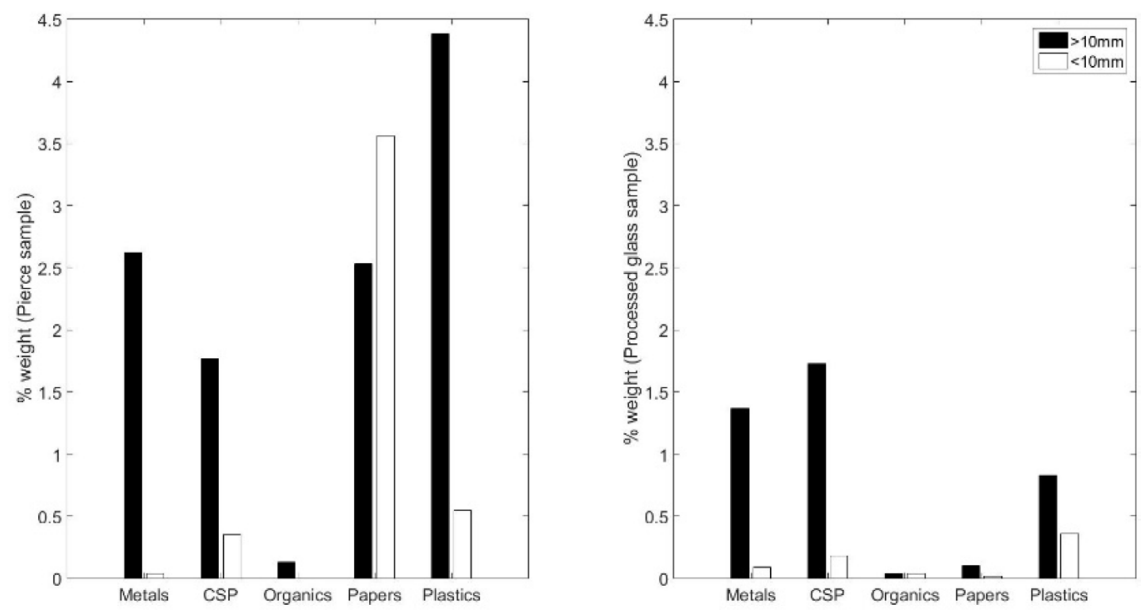

Figure 7: Contaminants composition on Site 1 feedstock before (left) and after (right) the separation process. Mean values of five independent samples.

The pressure drop considered for the experiments carried in the full scale rig is 6 bar. However, the incidence angle of the hydrofoils is modified from positive to negative angle of attack.

When the hydrofoils are position with positive angle of attack small particles of glass and sand are stacked up in them, reducing their efficiency. Therefore, the hydrofoils are positioned at negative angle of attack, as depicted in Fig. 6, before starting the runs that are recorded.

\section{RESULTS}

The results are evaluated in terms of weight content of contaminants in the glass concentrate compared with the quality of the initial product. The procedure followed to identify the quality of the glass cullet and the size distribution is the same as the one exposed in above.

The contaminants present in the glass cullet samples are greatly reduced when compared with the raw product, as shown in Fig. 7. Plastics and papers are the contaminants that are better removed when particles bigger than $10 \mathrm{~mm}$ are considered, having a reduction of $96.1 \%$ in papers and $81.1 \%$ in plastics.

However, for particles smaller than $10 \mathrm{~mm}$, plastic contaminants are reduced in $35.3 \%$ while papers and CSP are greatly reduced in $99.5 \%$ and $49.6 \%$, respectively. Metal and organic contaminants greater than $10 \mathrm{~mm}$ also exhibit an interesting reduction of $47.6 \%$ and $73.4 \%$, respectively.

A de-sludge is performed after processing 87.14 tonnes of MRF. Although a significant amount of sludge is likely to remain in the lamellas, a volume of $0.84 \mathrm{~m}^{3}$ of thick sludge is 

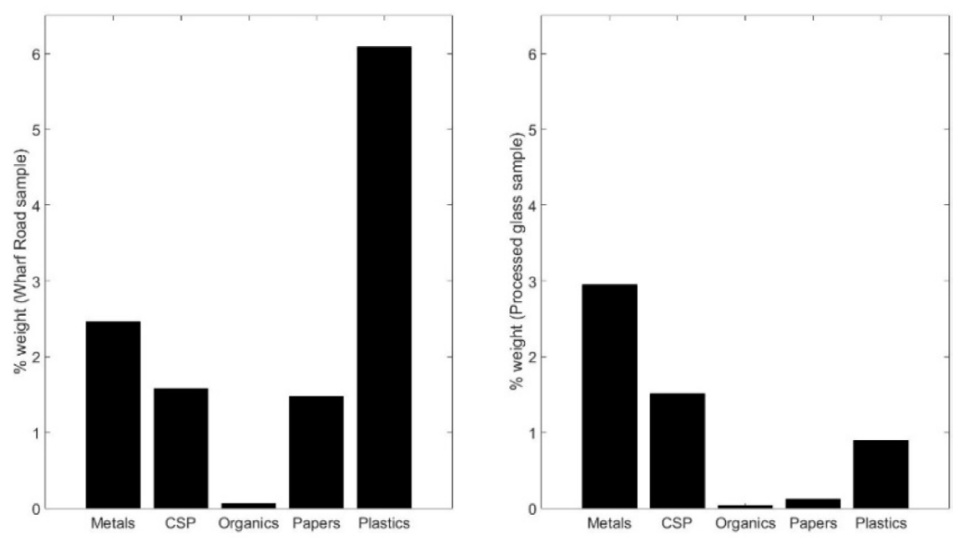

Figure 8: Contaminants composition on Site 2 feedstock before (left) and after (right) the separation process.

obtained. Tests carried by Derwentside Environmental Testing Services (DETS) will define the composition of the sludge recovered.

The particle size distribution in Table 3 proves that there is not a significant change between the input and the output glass particle size, fact that will benefit the latter glass treatment.

In order to do verify the robustness of the separation process, a quick analysis is carried with feedstock from Site 2. This new product has a higher level of contaminants compared with the former, being the high metal content the most remarkable characteristic. The overall results of the separation process are exposed in Fig. 8. The separation of metal contaminants is not of high importance since a metal removal process will be carried prior to entering the separator.

Conversly, plastic particles are reduced $85.2 \%$ of its original content while papers removal reach the figure of $91.9 \%$.

\section{CONCLUSION}

This novel hydrodynamic separator represents a new approach to separate particles of a wide range of size and density based on their Stokes number. Although the percentage of contaminants in the clean glass product is sufficiently small for the glass recycling industry, this technique could be applied at industrially relevant flow of around 10 tonnes per hour to prepare the feed of the optical sorters in the glass recovery facilities, too. The cleanness of the final product would greatly simplify the optical sorter stage in case this separation process is required.

Using feedstock from Site 1, it was possible to reduce significantly the content of contaminants in a single-stage separation process. Although it has been shown that small particles of plastic were not as effectively removed as the big ones, the removal rate is still acceptable. Based on the time scale theory, small plastics may have similar characteristic time scales to glass particles, making them more difficult to distinguish in terms of turbulence interaction.

The hydrodynamic separator is verified as a robust device capable of dealing with highly contaminated feedstock, i.e. Site 2. A more detailed investigation will be carried to establish 
the limitations of this device in terms of the contaminant sizes that can be effectively removed. We are currently investigating the turbulence structures in the flow to have a better insight of the separation process and to further increase the separation efficiency.

\section{REFERENCES}

[1] Study on total waste management of Jimma southern Ethiopia, LEM: the Environment \& Development Society of Ethiopia, 2006.

[2] Williams, M., Waste-to-energy success factors in Sweden and the United States. Analyzing the transferability of the Swedish waste-to-energy model to the United States, 2011.

[3] Digest of Waste and Resource Statistics - 2015 Edition, U.K. Department for Environment Food \& Rural Affairs. PB14292 Online, availbale at https://www.gov.uk/ government/uploads/system/uploads/attachment_data/file/482255/Digest_of_waste_ England_-_finalv3.pdf

[4] Hoerner, S., Fluid-Dynamic Drag, 2nd edn., Published by the author: Midland Park, N.J, 1965.

[5] Hölzer, A. \& Sommerfeld, M., New simple correlation formula for the drag coefficient of non-spherical particles. Powder Technology. 184, pp 361-365, 2008.

http://dx.doi.org/10.1016/j.powtec.2007.08.021

[6] Gabito, J. \& Tsouris, C., Drag coefficient and settling velocity for particles of cylindrical shape. Powder Technology, 183, pp. 314-322, 2008.

http://dx.doi.org/10.1016/j.powtec.2007.07.031

[7] Zhang, H., Ahmadi, G., Fan, F. \& McLaughlin, J., Ellipsoidal particles transport and deposition in turbulent channel flows. International Journal of Multiphase Flow, 27(6), pp. 971-1009, 2001.

http://dx.doi.org/10.1016/S0301-9322(00)00064-1

[8] Stringham, G.E., Simons, D.B. \& Guy, H.P., The behaviour of Large Particles Falling in Quiescent Liquids. Sediment Transport in Alluvial Channels. Geological Survey Professional Paper 562-C, 1969.

[9] Fornari, W., Picano, F., \& Brandt, L., Sedimentation of finite-size spheres in quiescent and turbulent environments. Journal of Fluid Mechanics, 788, pp. 640-669, 2016. http://dx.doi.org/10.1017/jfm.2015.698

[10] Yang, C.Y. \& Lei, U., The role of turbulent scales in the settling velocity of heavy particles in homogeneous isotropic turbulence. Journal of Fluid Mechanics, 371, pp. 179-205, 1998.

http://dx.doi.org/10.1017/S0022112098002328

[11] Tanaka, T. \& Eaton, J.K., Sub-Kolmogorov resolution particle image velocimetry measurements of particle-laden forced turbulence. Journal of Fluid Mechanics, 643, pp. 177-206, 2010. http://dx.doi.org/10.1017/S0022112009992023

[12] Yang, T.S. \& Shy, S.S., The settling velocity of heavy particles in an aqueous near-isotropic turbulence. Physics of Fluids, 15(4), pp. 868-880, 2003. http://dx.doi.org/10.1063/1.1557526

[13] Gore, R.A. \& Crowe, C.T., Effect of particle size on modulating turbulent intensity. International Journal of Multiphase Flow, 15(2), pp. 279-285, 1989. http://dx.doi.org/10.1016/0301-9322(89)90076-1 
[14] Elghobashi, S. \& Truesdell, G.C., On the two-way interaction between homogeneous turbulence and dispersed solid particles. I: Turbulence modification. Physics of Fluids, 5, pp. 1790-1801, 1993. http://dx.doi.org/10.1063/1.858854

[15] Balachandar, S. \& Eaton, J.K., Turbulent dispersed multiphase flow. Annual Reviews Fluid Mechanics, 42, pp. 111-133, 2010. http://dx.doi.org/10.1146/annurev.fluid.010908.165243

[16] Aquavitrum Ltd, CDPA 1988 patent no: GB2512270 \& patent pending no: GB1412552.0, 2013.

[17] Pope, S.B., Turbulent Flows, Cambridge University Press, 2000. http://dx.doi.org/10.1017/CBO9780511840531 